\title{
Coccidioidomycosis in Chicken Pullets in Jos, Plateau State, Nigeria: A Case Report
}

\author{
JAMBALANG ${ }^{1 *}$, A.R., OGO', I.N., IBU ${ }^{1}$, J.O., GISILANBE ${ }^{1}$, M., BERTU ${ }^{1}$, W., JWANDER ${ }^{1}$, L., \\ BENJAMIN ${ }^{1}$, B., CHUKWUKERE ${ }^{1}$, S., NASIR ${ }^{1}$, Y., SANDA ${ }^{1}$, N., BENSHAK ${ }^{1}$, A., AGADA ${ }^{1}$, G.O.A \\ and $\mathrm{KUBO}^{2}, \mathrm{M}$.
}

${ }^{1}$ National Veterinary Research Institute Vom, Plateau State, Nigeria ${ }^{2}$ National Institute of Animal Health, Tsukuba, Ibaraki, Prefecture, Japan. *Corresponding author: drjambalang@yahoo.com, TEL. +234 7065317325

\section{INTRODUCTION}

Coccidioidomycosis is a dust-borne infection caused by a dimorphic fungus Coccidioides immitis and is known to occur in man and a wide variety of wild and domesticated animals including deers, mice, rats, monkeys, dogs, cattle, and horses, but have not been shown to infect birds (Merck Veterinary Manual, 1998; Saubolle et al., 2007). The organism grows well in cultures with recovery rate of $0.4 \%, 2.6 \%$ and $8.3 \%$ respectively for blood, bone marrow and respiratory tract cultures respectively (Saubolle et al., 2007). They produce aerial mycelia which form a small, fluffy-white, spherical colony; in tissues the fungus takes the form of spherules (sporangia) 20-80 pm with double contoured walls (Jones et al., 1997; Kaufman and Standard, 1987). The disease occurs mainly in the Americas where farmers and others who work with soil are most likely to inhale the spores and become infected.

Coccidioidomycosis in animals usually assumes the chronic progressive form with primary lesions in the lungs and disseminate initially into regional lymph nodes, then to almost any internal organ within the chest or abdomen. In some species like the dog and monkey, lesions may be disseminated throughout the skeletal system and also present with ocular involvement (Jones et al., 1997; Shubitz, 2007). Generalized infection usually runs a slow course; the signs are non specific and may include emaciation, in appetence, low-grade fever and occasional cough.

The gross lesions of coccidioidomycosis resemble those of tuberculosis in many respects; they appear as discrete or confluent granulomas with or without suppuration or calcification, in the disseminated form of the disease, grayish nodules of various sizes may be found in the lungs, lymph nodes, liver, spleen and other organs (Bharucha et. al., 1996; Merck Veterinary Manual, 1998). The nodules are usually irregular in shape and may exude material when squeezed. The microscopic appearance is characteristic, but varies to some extent in relationship to the developmental stage of the fungus; however, Kaufman et al. (1998) described some unusual morphologic forms of this organism in biopsy tissues taken from the lungs of a human patient. The spherules are often filled with endospores (sporangiospores) 2-5um in diameter and have wide zone of epithelial cells admixed with a few neutrophils and some lymphocytes. When the wall of a spherule ruptures, it releases a lot of endospores and the surrounding tissues express reactions that are rich in neutrophils and lymphocytes with few epithelioid cells (Saoubolle, 2007). The organisms within the cytoplasm of Langhans' giant cells are clearly seen in sections stained with hematoxylin and eosin. In the liver, spleen and lungs, the lesions are usually spherical and sharply circumscribed and expand to displace normal tissues (Jones et al., 1997; Merck Veterinary Manual, 1998). Microscopic demonstration of the organism (typical mature spherules filled with endospores or arthroconidia) in characteristic lesions is diagnostic.

In this report, the first recorded case of coccidioidomycosis in chickens in Nigeria diagnosed by postmortem analysis at the National Veterinary Research Institute, (NVRI) Vom Plateau State, Nigeria and confirmed by histopathology of lung tissues at the National Institute of Animal Health (NIAH), Tsukuba, Ibaraki Prefecture, Japan is presented.

KEY WORDS: Coccidioides immitis, Histopathology, pullets, Vom, Nigeria.

\section{CASE REPORT.}

Two 12- week old moribund pullets were presented to the Central Diagnostic Laboratory, NVRI, Vom Plateau State, Nigeria for postmortem analysis. Previously, 13 birds had died from the same flock of 
approximately 2,000 chickens two weeks prior to presentation of the case. History further revealed that the flock had all vaccinations according to industry standard prescribed for poultry vaccination schedule in Nigeria.

\section{Post mortem findings:}

Lesions observed at postmortem were; a slightly congested mucosal surface of the trachea, lungs which had whitish nodules, were congested and adhered to the thoracic cage. The serosal surface of the proventriculus was covered with whitish nodular substances. The liver was enlarged $\left(1 \frac{112}{X)}\right.$ and covered with the same whitish nodular substances seen on the epicardium, lungs and the proventriculus. The examination of the gastrointestinal tract revealed hemorrhage on the caecal tonsil and intestine with sloughing of the mucosal surface. The following organs were enlarged; sciatic nerves, kidneys, and the Bursa of Fabricius, while the brain was congested and the spleen discolored.

Samples from various organs (trachea, lungs, liver, spleen, proventriculus and gall bladder) were collected aseptically and subjected to viral, bacterial, and parasitological examination at the National Veterinary Research Institute (NVRI), Vom Plateau State, Nigeria. It is pertinent to say that mycological examination was not carried out at this stage due to nonavailability of diagnostic materials required for such study. A tentative diagnosis of mycosis was made based on the number of mortality, history of the flock and postmortem lesions, while Marek's disease and coccidiosis were considered as differentials. Wax-embeded lung tissue samples were sent to the National Institute of Animal Health (NAIH), Tsukuba, Ibaraki Prefecture, Japan, for confirmatory diagnosis. Various staining techniques (Haematoxylin and Eosin, Warthin Starry, and Grocott Methenamine Silver) were used to confirm the presence of the causative organism Coccidioides immitis in lung tissues. Figure 1 shows lung tissues stained with Haematoxylin and Eosin displaying spherules with endospore of $C$. immitis. Figures 2 and 3 show spherules, endospores and mycelia of C. immitis in different stains as well asn under fluorescent microscope. The presence of mycelia in the lung tissues goes a long way to substantiate the work of Saubolle, (2007) in which he asserted that mycelia can be found in the lungs, skin and ventricular fluids.

The presence of spherules with double contoured walls, alongside the endospores, and the absence of budding as observed in this case helped to confirm the presence of the organism in the lung tissues. The above characteristics are normally used to distinguish Coccidioides immitis from other mycosis.

Oocysts of Eimeria spp ( $<100$ opg) were observed from the intestinal scrapings, no other organism was isolated during viral and bacterial analysis.

\section{DISCUSSION AND CONCLUSION}

Coccidioidomycosis, a non-contagious endemic disease seen mainly in the Western part of the United States and South America is acquired by inhalation of the spores of Coccidioides immitis and is diagnosed by direct microscopic examination of endospore-containing spherules in infected materials amongst other arrays of diagnostic methods. In our report, the diagnosis of coccidioidomycosis in chicken was achieved by postmortem lesions on the internal organs as well as by histopathology and specific fluorescent antibody reaction of lung tissues which had most of the whitish nodular lesion and is known to support the growth of Coccidioides immitis.

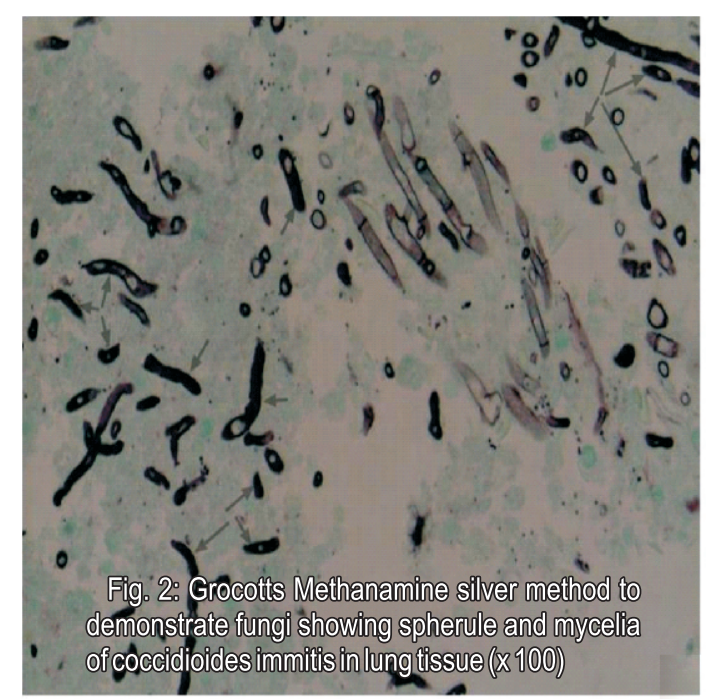




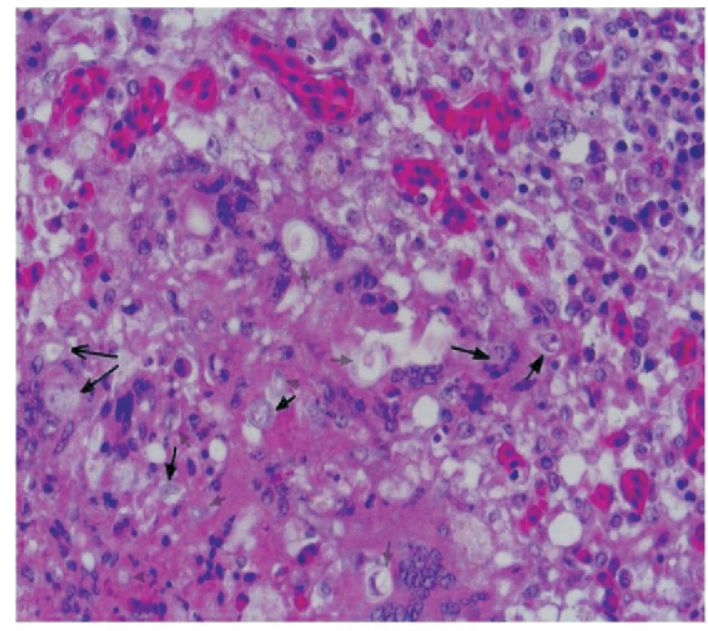

Fig. 1: H \& E Section showing spherule (arrows) of Coccidioides immitisIn lung tissue (X100)

The microscopic examination of these nodules using the various stains, most especially the highly sensitive and specific Grocott Methenamine Silver stain revealed several spherules with endospore as well as mycelia all of which are common in infected animal hosts and are considered to be diagnostic for the disease (Kirkland and Fierer, 1996: Saubolle, 2007).

The occurrence of Coccidioidomycosis in chicken is uncommon in this part of the world and in Nigeria in particular, and as such this diagnosis is of utmost importance considering the rarity of the infection in avian species, although it is capable of infecting all mammals and reptiles, but not any avian species to date (Timm, et al., 1988; Shubitz, 2007).

Coccidioidomycosis infection is of public health significance in the light of the zoonotic nature of the disease, and because the effective control of many human zoonoses requires control in animals, it is imperative that farmers, veterinarians, and all personnel involved in the poultry industry who are at a higher risk of this infection always wear well fitted masks when handling any poultry.

\section{REFERENCES:}

BHARUCHA, N. E., RAMAMOORTHY, K., SORABJEE, J., and KURUVILLA, T. (1996): All that caseates is not tuberculosis. Lancet. 348 (9037): 1313.

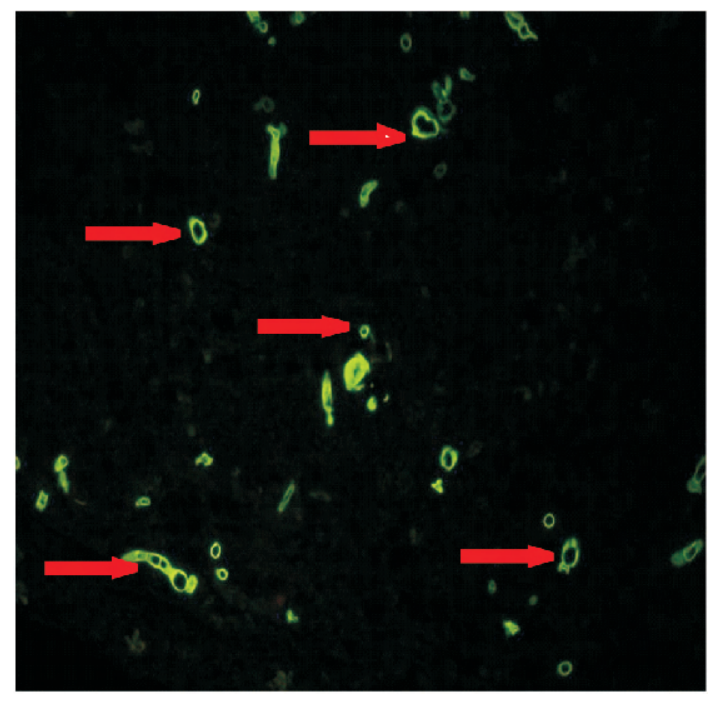

Fig. 3: Warthin Starry (3) Stain showing spherules and endospores of coccidioides immitis in lung

JONES, T. C., HUNT, R. D., and KING, N.W. (1997) Veterinary Pathology, sixth edition, Lippincott, Williams \& Wilkins: 510-512.

KAUFMAN, L. and STANDARD, P. G. (1987): Specific and rapid identification of medically important fungi by exoantigen detection. Annu. Rev. Microbiol. 41: 209-225.

KAUFMAN, L., VALERO, G., and PADHYE, A.A. (1998): Misleading manifestations of Coccidioides immitis in vivo. J. Clin. Microbiol.36(12): 3721-3723.

KIRKLAND, T. N. and FIERER, J. (1996): Coccidioidomycosis: a reemerging infectious disease. Clinical characteristics and current controversies. Medicine. 83: 149-175.

MERCK VETERINARY MANUAL. (1998): Eight edition: Merck \& Co., Inc. Whitehouse station, N. J., USA: 2305pp.

SAUBOLLE, M.A. (2007): Laboratory aspects in the diagnosis of Coccidioidomycosis. Ann. N. Y. Acad. Sci. 1111:301-314.

SAUBOLLE, M.A., McKELLER, P.P., and SUSSLAND, D.(2007): Epidemiologic, Clinical and Diagnostic Aspect of Coccidioidomycosis. J. Clin. Microbiol. 45(1): 26-30.

SHUBITZ, L.A. (2007): Comparative aspects of Coccidioidomycosis in animals and humans. Ann. N.Y. Acad. Sci. 1111: 395403.

TIMM, K.I., SONN, R.J. and HULTGREN, B.D. (1988): Coccidioidomycosis in a Sonoran gopher snake (Pituophis melanoleucus affinis). J. Med. Vet. Mycol. 26: 101-104. 